\title{
Role of NASA 10-minute Lean Test in diagnosing postural orthostatic tachycardia syndrome: a preliminary study in young population
}

\author{
Musharaf Bashir, ${ }^{1}$ Himani Ahluwalia, ${ }^{2}$ Tazeen Khan, ${ }^{1}$ Sheikh Imran Sayeed ${ }^{1}$ \\ ${ }^{1}$ Department of Physiology, GMC \& SMHS Hospital, Srinagar, J\&K; ${ }^{2}$ Department of Physiology, VMMC \& Safdarjung Hospital, \\ New Delhi, India
}

\begin{abstract}
Postural orthostatic tachycardia syndrome (POTS) is a form of autonomic imbalance characterized by abnormally increased heart rate (HR) and various symptoms of orthostatic intolerance (OI). This study aimed to determine POTS in adolescents using the National Aeronautics and Space Administration (NASA) 10-minute Lean Test. This cross-sectional study was conducted at Government Medical College, Srinagar, J\&K, India, between October 2017 and November 2019. Adolescents with symptoms of OI were enrolled for this study, and the NASA 10-minute Lean Test was performed. Characteristics of the POTS group were compared with the non-POTS group. There was a significant difference in BMI $(\mathrm{P}<0.0001)$ between the POTS and non-POTS groups. However, the number of females with features of POTS was greater than males. There was a significant difference in resting HR between the two groups $(\mathrm{P}<0.0001)$. During upright posture, there was a significant difference in maximum upright HR between the two groups $(\mathrm{P}<0.0001)$, the highest increment in upright $\mathrm{HR}$ also differed significantly (P<0.0001). POTS group had significantly higher HR than the non-POTS group. It may be inferred from this preliminary study that NASA 10minute Lean Test can be used to diagnose POTS.
\end{abstract}

\section{Introduction}

Postural orthostatic tachycardia syndrome (POTS) is a form of cardiovascular autonomic im-

Correspondence: Musharaf Bashir, Department of Physiology, GMC \& SMHS hospital, Srinagar, J\&K, India.

Tel.: +91.7042272380 .

E-mail: dr.musharaf.mb@gmail.com

Key words: Postural orthostatic tachycardia syndrome; adolescents; Lean Test; NASA.

Acknowledgments: the authors are very grateful to Dr. Himani Ahluwalia and Dr. Raj Kapoor and all faculty of the Department of Physiology, VMMC \& Safdarjung Hospital, New Delhi, India, for their valuable suggestions and moral support.

Contributions: MB, manuscript preparation, data collection; HA, data collection, manuscript revision; TK, statistical analysis, literature; SIS, manuscript revision.

Conflict of interests: the authors declare no potential conflict of interests.

Received for publication: 20 June 2020.

Revision received: 29 December 2020.

Accepted for publication: 30 December 2020.

This work is licensed under a Creative Commons Attribution NonCommercial 4.0 License (CC BY-NC 4.0).

${ }^{\circ}$ Copyright: the Author(s), 2021

Licensee PAGEPress, Italy

Italian Journal of Medicine 2021; 15:85-89

doi:10.4081/itjm.2021.1340 balance defined by an increased resting heart rate with features of chronic orthostatic intolerance (OI).$^{1,2}$ In adolescents, POTS is characterized by an increase in heart rate (HR) $\geq 40$ beats/minute (bpm) from the resting HR values in the first 10 minutes upon assuming an upright posture or if the HR remains $\geq 120 \mathrm{bpm}$ in a supine position. ${ }^{3}$ It is accompanied by symptoms of OI such as lightheadedness, nausea, shortness of breath, diaphoresis, headache, fatigue, cognitive dysfunction usually described as brain fog, and other features of autonomic imbalance which sometimes may be severe enough to decrease the quality of life. ${ }^{2,4}$ Typically orthostatic hypotension $(\mathrm{OH})$ is not seen in POTS. ${ }^{3}$ POTS is seen in all age groups, although it is more common in the young population, especially in premenopausal females with an overall prevalence of $0.2-1 \%{ }^{5}$ In adolescents, POTS has been seen to occur at a rate of $6.8 \%$, affecting their quality of life. ${ }^{6}$ POTS has a multifactorial etiology such as catecholamine excess, autoimmune disorders, hypovolemia, vaccination, infection, and pregnancy. ${ }^{1}$ In clinical settings, the tilttable test remains the gold standard for the diagnosis of POTS. ${ }^{7}$

To the best of our knowledge, this is the first study to use the NASA 10-minute Lean Test, which is regarded as equivalent to the tilt-table test ${ }^{8}$ for the diagnosis of POTS in adolescents. Therefore, this study was aimed to see whether the NASA 10minute Lean Test can be used to diagnose POTS in adolescents. 


\section{Materials and Methods}

This observational cross-sectional study was conducted in the Department of Physiology at GMC \& SMHS Hospital, Srinagar, J\&K, India, between October 2017 and November 2019 after obtaining approval from the Institutional Ethical Committee. In this study, 200 adolescent subjects, aged 12 to 19 years, were enrolled. They filled a questionnaire to report the symptoms of OI. Out of 200 subjects, 150 reported symptoms of OI. Eventually, 150 subjects with symptoms of OI were selected for this study. Participants who had a history of smoking, chronic diseases, infectious diseases, head trauma, diabetes mellitus, anemia, psychiatric disorders, and intake of drugs affecting autonomic functions were excluded from this study by taking a thorough history. OI was assessed with the help of an Orthostatic Intolerance Questionnaire $(\mathrm{OIQ})^{9}$ which was filled by all the subjects. They were asked to report to the autonomic function testing laboratory in the Department of Physiology, at the GMC $\&$ SMHS Hospital. There, they were explained the protocol of the NASA 10-Minute Lean Testing in detail; such as limiting fluid and sodium intake at least 24 hours before testing, not to wear compression socks, and withhold medicine that might influence autonomic functions such as $\beta$ blockers, Midodrine, fludrocortisone, antidepressants, and caffeine. The test was performed between 9:00 and 11:00 a.m., and standardized conditions were maintained throughout the procedure. Anthropometric measurements such as height, weight, and BMI were calculated. After that, all the participants were rested in a supine position for at least 10-15 minutes to achieve hemodynamic equilibrium. After 15 minutes of rest in the supine position, systolic blood pressure (SBP, $\mathrm{mmHg}$ ), diastolic blood pressure $(\mathrm{DBP}, \mathrm{mmHg})$, and $\mathrm{HR}$ were recorded with the help of a digital sphygmomanometer (OMRONHEM-7120, Kyoto, Japan). Vitals were again measured 1 minute later. Readings were retaken in case vitals were not similar until two consecutive readings were almost the same. This was done to determine true resting vitals. After the procedure mentioned above, participants were asked to stand up and walk to a specified wall and lean against the wall in such a way that only their shoulder blades touched the wall without arching the lower back. To avoid the contact of buttocks and wall, subjects were asked to place the heels at a specified mark that was 6 inches away from the wall (Figure 1). The test began once the subject leaned comfortably against the wall. After 1 minute of passive standing, SBP, DBP, and HR were recorded. The whole procedure was repeated every minute for the next 9 minutes. At the end of every minute, subjects were told to report symptoms of OI, if any. The NASA 10- minute Lean Test procedure was demonstrated to each subject before the actual commencement of the test. Written consent was obtained from all participants or their caretakers, and the study was clarified beforehand. Participants had the choice to withdraw from the study at any time.

Data were analyzed using GraphPad Prism Version 6 (GraphPad Software, San Diego, CA-92108, USA). The normality of various parameters was determined using D'Agostiono-Pearson omnibus test. Chi-square test with Yates correction was used for variables. An unrepaired t-test was used to compare the two groups and the non-parametric data Mann-Whitney U test. Data were expressed in mean \pm standard deviation. Pvalue of $<0.05$ was considered statistically significant.

\section{Results}

A total of 200 subjects were enrolled in this study, and 150 out of them reported symptoms of OI filling out a questionnaire. Eventually, 150 subjects were en-

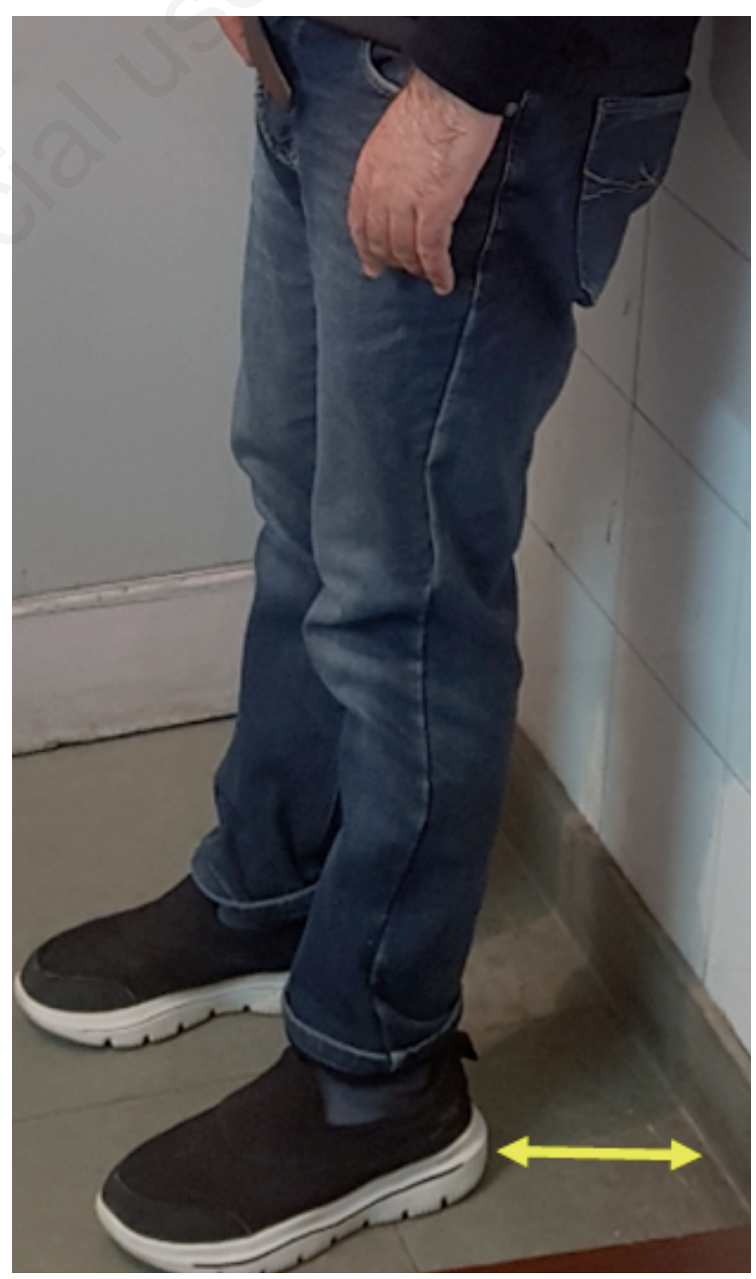

Figure 1. Passive lean posture during NASA 10-minute Lean Test. The feet should be placed straight and approximately 6 inches from the wall. 
rolled for this study. NASA 10-minute Lean Test was performed in all the subjects to assess OI and POTS. During the NASA 10-minute Lean Test, the most common symptom reported was lightheadedness/dizziness (61.3\%), which was followed by weakness $(52.7 \%)$, tremulousness (46.7\%), palpitations (46\%), blurring of vision $(41.3 \%)$, shortness of breath (38\%), chest pain $(30 \%)$, increased sweating (19.3\%) and presyncope/syncope (13.3\%) (Table 1). It was observed that 40 participants fulfilled the criteria of POTS using this test. Characteristics of the POTS group were compared with the 110 of non-POTS group. There was no significant difference in age $(16.29 \pm 2.64$ years $v s$ $17.23 \pm 1.31$ years; $\mathrm{P}=0.381)$ and gender $\left(\chi^{2}=0.679\right.$; $\mathrm{P}=0.410$ ) between the two groups. BMI of the POTS group was significantly lower than non-POTS group $\left(18.50 \pm 0.53 \mathrm{~kg} / \mathrm{m}^{2}\right.$ vs $\left.21.38 \pm 0.33 \mathrm{~kg} / \mathrm{m}^{2} ; \mathrm{P}<0.0001\right)$ (Table 2). After a supine rest of about 10-15 minutes, systolic blood pressure (SBP), diastolic blood pressure (DBP), and heart rate (HR) were recorded. There was no significant difference between resting SBP (117.7 $\pm 9.38 \mathrm{mmHg}$ vs $118 \pm 10.47 \mathrm{mmHg} ; \mathrm{P}=0.823$ ) and resting DBP $(72.71 \pm 7.45 \mathrm{mmHg}$ vs $73.80 \pm 7.91$ $\mathrm{mmHg} ; \mathrm{P}=0.657$ ) between the two groups. However, the resting HR of the POTS group was significantly higher (93.94 \pm 16.89 bpm vs $75.66 \pm 9.29$ bpm; $\mathrm{P}<0.0001$ ) (Table 2). In the supine position, there was no significant difference between the average SBP (116 $\pm 10.11 \mathrm{mmHg}$ vs $115 \pm 9.16 \mathrm{mmHg} ; \mathrm{P}=0.531)$ and average DBP $(72.67 \pm 8.21 \mathrm{mmHg} v s 72.91 \pm 8.72 \mathrm{mmHg}$; $\mathrm{P}=0.814$ ) between the two groups. The average HR was significantly higher in the POTS group (92.61 \pm 15.32 bpm vs $75.72 \pm 9.01$ bpm; $\mathrm{P}<0.0001)$ (Table 2). During the lean test, vitals were recorded at the end of each minute for 10 minutes, and average SBP and DBP were calculated. There was no significant difference in average SBP $(112 \pm 10.11 \mathrm{mmHg} v s$ $115 \pm 9.16 \mathrm{mmHg} ; \mathrm{P}=0.531)$ and $\mathrm{DBP}(70.81 \pm 11.21$ $\mathrm{mmHg} v s$ 71.10 \pm 9.73 ; $\mathrm{P}=0.645)$ of 10 minutes between the two groups. It was observed that the highest rise in HR (133.6 $\pm 14.27 \mathrm{bpm} v s 91.67 \pm 9.94 \mathrm{bpm}$; $\mathrm{P}<0.0001)$ and the maximum increase in HR $(40.91 \pm 11.78$ bpm vs $16.34 \pm 6.97 \mathrm{bpm} ; \mathrm{P}<0.0001)$ were significantly higher in the POTS group (Table 2).

\section{Discussion}

POTS is a form of dysautonomia characterized by an exaggerated $\mathrm{HR}$ and various symptoms of $\mathrm{OI}$ in the

Table 1. Symptoms of orthostatic intolerance in decreasing order as assessed by NASA Lean Test.

\begin{tabular}{lc}
\hline Orthostatic symptoms & $\begin{array}{c}\text { Frequency (\%) } \\
\mathbf{N = 1 5 0}\end{array}$ \\
\hline Light headedness/dizziness & $92 / 150(61.3 \%)$ \\
\hline Weakness & $79 / 150(52.7 \%)$ \\
\hline Tremulousness & $70 / 150(46.7 \%)$ \\
\hline Palpitations symptoms & $69 / 150(46 \%)$ \\
\hline Blurring of vision & $62 / 150(41.3 \%)$ \\
\hline Shortness of breath & $57 / 150(38 \%)$ \\
\hline Chest pain & $45 / 150(30 \%)$ \\
\hline Increased sweating & $29 / 150(19.3 \%)$ \\
\hline Pre-syncope/syncope & $20 / 150(13.3 \%)$ \\
\hline
\end{tabular}

NASA, National Aeronautics and Space Administration. N, total number of subjects.

Table 2. Comparison of characteristics between Type-1 diabetics with postural orthostatic tachycardia syndrome vs nonpostural orthostatic tachycardia syndrome.

\begin{tabular}{|c|c|c|c|c|}
\hline Characteristics & $\begin{array}{c}\underset{n=40}{\text { POTS with OI }} \\
\quad n=4\end{array}$ & $\begin{array}{c}\begin{array}{c}\text { Non-POTS with OI } \\
n=110\end{array}\end{array}$ & $\chi^{2}$ & P value \\
\hline Female:Male & $31: 08$ & $100: 20$ & 0.679 & 0.410 \\
\hline Age (yrs) & $16.29 \pm 2.64$ & $17.23 \pm 1.31$ & & 0.381 \\
\hline BMI $\left(\mathrm{kg} / \mathrm{m}^{2}\right)$ & $18.50 \pm 0.53$ & $21.38 \pm 0.33$ & & $<0.0001 * *$ \\
\hline \multicolumn{5}{|l|}{ Supine position } \\
\hline Resting SBP (mmHg) & $117.7 \pm 9.38$ & $118 \pm 10.47$ & & 0.823 \\
\hline Resting DBP (mmHg) & $72.71 \pm 7.45$ & $73.80 \pm 7.91$ & & 0.657 \\
\hline Resting HR (bpm) & $93.94 \pm 16.89$ & $75.66 \pm 9.29$ & & $<0.0001 * *$ \\
\hline Average SBP (mmHg) & $116 \pm 10.11$ & $115 \pm 9.16$ & & 0.531 \\
\hline Average DBP (mmHg) & $72.67 \pm 8.21$ & $72.91 \pm 8.72$ & & 0.814 \\
\hline Average HR (bpm) & $92.61 \pm 15.32$ & $75.72 \pm 9.01$ & & $<0.0001 * *$ \\
\hline \multicolumn{5}{|l|}{ Passive standing (Lean test) } \\
\hline Average SBP $(\mathrm{mmHg})$ of 10 minutes & $112 \pm 10.11$ & $115 \pm 9.16$ & & 0.531 \\
\hline Average DBP $(\mathrm{mmHg})$ of 10 minutes & $70.81 \pm 11.21$ & $71.10 \pm 9.73$ & & 0.645 \\
\hline Highest HR (bpm) & $133.6 \pm 14.27$ & $91.67 \pm 9.94$ & & $<0.0001^{* *}$ \\
\hline Highest increment in HR (bpm) & $40.91 \pm 11.78$ & $16.34 \pm 6.97$ & & $<0.0001 * *$ \\
\hline
\end{tabular}

All the data were expressed as mean \pm standard deviation. POTS, postural orthostatic tachycardia syndrome; OI, orthostatic intolerance; $\chi^{2}$, Chi-square test; n, number of subjects; BMI, body mass index; SBP, systolic blood pressure; DBP, diastolic blood pressure; HR, heart rate. ** Data is very highly significant. 
absence of OH. ${ }^{10,11}$ POTS can occur in any age group; however, it is more common in people aged 15-50 years, predominantly in females compared to males with a ratio of $4: 1$, which may be attributed to the dilation of vessels caused by female sex hormones. ${ }^{12,13}$ Symptoms of OI such as lightheadedness, chest pain, blurring of vision, and palpitations are usually seen in POTS. These symptoms arise due to decreased venous return, reduced cardiac output, and hypoperfusion. ${ }^{13}$ POTS has an abrupt onset with a multifactorial etiology and negatively impacts quality of life, so its timely diagnosis is important, especially in the young population. ${ }^{13,14}$ POTS is commonly diagnosed in clinical settings by a head-up tilt table test (HUTT), which is considered the gold standard for its diagnosis. ${ }^{1,5} \mathrm{In}$ this study, a simple, bedside, and passive standing test called NASA 10-minute Lean Test was used to evaluate POTS. NASA researchers used this test to assess OI symptoms in astronauts. Since it is a passive test, it reduces the muscular impact on venous return. This test has been ratified as an equivalent measure of OI as compared to HUTT. ${ }^{8}$

To the best of our knowledge, this is the first study to use the NASA 10-minute Lean Test to diagnose POTS in adolescents who had symptoms of OI.

Literature shows that POTS can cause decreased appetite in humans, leading to increased sympathetic activity and muscle wasting, thus causing lower BMI in them. ${ }^{11}$ Lin et al. in a study, compared the BMI of 111 children and adolescents having POTS with BMI of 154 children and adolescents having vaso-vagal syncope (VVS) and 82 healthy controls. They observed that BMI in the POTS group was significantly lower (18.3 \pm 3.4$)$ than in VVS (20 \pm 4.2$)$ and the control group [(20.5 \pm 2.9$) ; \mathrm{P}<0.05] .{ }^{15}$ Findings in our study were similar. This study observed no significant difference between the resting BP of the two groups; however, resting HR in the POTS group was significantly higher, which may be a result of autonomic imbalance characterized by increased cardiac sympathetic activity. Roernik et al. made similar observations in a study in which they demonstrated that HR in the POTS group was significantly higher than the non-POTS group [71 $711 \mathrm{bpm} v s \quad 65 \pm 9 \mathrm{bpm}$; $\mathrm{P}=0.009] .{ }^{16}$

During the NASA 10-minute Lean Test, no significant differences were observed between the upright BP of the two groups; however, upright HR was significantly higher in the POTS group. Upon assuming an upright posture, venous pooling and diminished cardiac output occur in patients with POTS ensuing reflex increase in HR. ${ }^{13}$ An increase in HR $\geq 40 \mathrm{bpm}$ from the resting levels upon assuming an upright posture or a resting HR $\geq 120 \mathrm{bpm}$ are characteristic of POTS. Lin et al. in a cross-sectional study, compared children with POTS vs non-POTS. They observed that the upright HR in the POTS group was significantly higher than the non-POTS group [32 $\pm 12 \mathrm{bpm} v$ s $22 \pm 7 \mathrm{bpm} ; \mathrm{P}<0.01]$, and they also observed that the resting HR in the POTS group was significantly higher than non-POTS group $[126 \pm 10 \mathrm{bpm}$ vs $107 \pm 11 \mathrm{bpm} ; \mathrm{P}<0.001]{ }^{15}$ This study observed that the maximum upright increment in HR in adolescent diabetics with POTS was significantly higher than the non- POTS group $(40.91 \pm 11.78 \mathrm{bpm} v s$ $16.34 \pm 6.97$ bpm; $\mathrm{P}<0.0001)$.

The current study has limitations. A major limitation is that NASA 10-minute Lean Test has not been used before, so there are very scarce data regarding this test. Another major limitation is that very few studies have been done to evaluate POTS, especially in the young population. Physiological POTS, which may be seen in some adolescents, was not ruled out. A small sample size may have caused variability in comparisons. Since this is a preliminary study, further studies using NASA 10-minute Lean Test are needed in the future to assess POTS.

\section{Conclusions}

Based on the observations of this preliminary study, it may be concluded that a simple, time-saving, and inexpensive Lean Test may be used to diagnose POTS in the young population.

\section{References}

1. Fedorowski A. Postural orthostatic tachycardia syndrome: clinical presentation, etiology and management. J Intern Med 2019;285:352-66.

2. Boris JR. Postural orthostatic tachycardia syndrome in children and adolescents. Auton Neurosci Basic Clin 2018;215:97-101.

3. Stewart JM, Boris JR, Chelimsky G, et al. Pediatric disorders of orthostatic intolerance. Pediatrics 2018;141:1.

4. Shaw BH, Stiles LE, Bourne K, et al. The face of postural tachycardia syndrome - insights from a large crosssectional online community-based survey. J Intern Med 2019;286:438-48.

5. Bryarly M, Phillips LT, Fu Q, et al. Postural orthostatic tachycardia syndrome. JACC 2019;73:1207-28.

6. Tao C, Wenxin L, Lin J, et al. Long term outcomes of children and adolescents with postural orthostatic tachycardia syndrome after conventional treatment. Front Pediatr 2019;7:00261.

7. McDonald C, Koshi S, Busner L, et al. Postural tachycardia syndrome is associated with significant symptoms and functional impairment predominantly affecting young women: a UK perspective. BMJ Open 2014;4: e004127.

8. Bateman L. Simple way to assess orthostatic intolerance; Bateman Horne Center. Available from: www. BatemanHorneCenter.org Accessed: Aug, 2017.

9. Moon J, Do-Yong K, Jung-Ick B, et al. Orthostatic intolerance symptoms are associated with depression and 
diminished quality of life in patients with postural tachycardia syndrome. BioMed Central 2016;14:144.

10. Singer W, Sletten DM, Opfer-Gehrking TL, et al. Postural tachycardia in children and adolescents: what is abnormal? J Pediatr 2012;160:222-6.

11. Kanjwal K, Karabin B, Kanjwal Y, Grubb BP. Autonomic dysfunction presenting as postural orthostatic tachycardia syndrome in patients with multiple sclerosis. Int J Med Sci 2010;7:62-7.

12. Anjum I, Sohail W, Hatipoglu B, Wilson R. Postural orthostatic tachycardia syndrome and its unsual presenting complaints in women: a literature minireview. Cureus 2018; 10:e2435.
13. Kavi L, Gammage MD, Karabin BL. Postural tachycardia syndrome: multiple symptoms, but easily missed. $\mathrm{Br}$ J Gen Pract 2012;62:286-87.

14. Pederson JL, Brook JB. Health related quality of life and suicide risk in postural tachycardia syndrome. Clin Auton Res 2017;27:75-81.

15. Lin J, Zhao H, Jiao F. Body mass index is decresed in children and adolescents with postural tachycardia syndrome. Turk J Pediatr 2019;61:52-8.

16. Roerink ME, Lenders JWM, Schmits IC, et al. Postural orthostatic tachycardia is not a useful diagnostic marker for chronic fatigue syndrome. J Intern Med 2017;282: 179-88. 